\title{
An Emergency Operation Model in Upstream Pools of the Accident Pool of the Middle Route of South-to-North Water Diversion Project
}

\author{
Jin Quan ${ }^{1}$, Hezhen Zheng ${ }^{2, a}$, Siyu Cai ${ }^{1}$, Yi Xu ${ }^{3}$ \\ ${ }^{1}$ Sate Key Laboratory of Simulation and Regulation of Water Cycle in River Basin, China Institute of Water Resources and Hydropower \\ Research, Beijing 100038, China \\ ${ }^{2}$ Changjiang Survey, Planning, Design and Research Co., Ltd. Wuhan 430010, China \\ ${ }^{3}$ College of Civil Engineering and Architecture, Zhejiang University, Hangzhou 310058, China
}

\begin{abstract}
Considering the potential emergency accident in the Middle Route of South-to-North Water Diversion Project (MRP), previous studies required upstream pools of accident pool where accident occurs to maintain constant downstream level in the final state of emergency operation, which could cause large amount of abandoned water. In order to save water resources, an emergency operation model in upstream pools of the accident pool of the MRP was built, which allows downstream levels to rise by a certain amount in the final state, and can minimize the total abandoned water through allocating volumes in the upstream pools. This model could play an important role to determine reasonable emergency operation measures in the upstream pools.
\end{abstract}

\section{Introduction}

Water shortage has become a problem of global concern that poses a substantial threat to food security, economic development and ecological health [1]. A promising way to solve the problem of uneven spatial and temporal distribution of water resources is the construction of water diversion projects [2]. Up to now, over 350 longdistance water diversion projects have been constructed in 39 countries worldwide [3]. In China, the most wellknown water diversion project is the Middle Route of South-to-North Water Diversion Project (MRP), which aims to divert water from Danjiangkou Reservoir in southern China to the arid northern China, including Henan, Hebei, Tianjin and Beijing [4].

The MRP passes through a lot of roads, bridges and is along with many chemical factories, making emergency accidents potential to occur, such as sudden water pollution accidents, which may endanger water supply safety and cause economic losses [5]. If serious emergency accident occurs, the main canal can be divided into the accident pool where the accident occurs, and pools upstream and downstream of the accident pool. The two check gates at both ends of the accident pool should be closed rapidly, the discharge of the diversion gates of upstream pools should remain unchanged, and the water supply of downstream pools should be maintained for a period of time using their own water volume [6]. Emergency operation of upstream pools is very difficult, and previous studies $[7,8]$ required that the upstream pools should be operated according to constant downstream level [9], which could make surplus volume abandoned and cause huge economic losses.

Our team briefly introduced an emergency operation model of upstream pools in a published paper [10], which allows downstream levels to rise by a certain amount in the final state to minimize the total abandoned water by allocating volumes in the upstream pools. The objective of this paper was to present this model in detail, which could help readers understand more clearly.

\section{Study area}

The MRP (Figure 1) is one of the largest inter-basin water diversion projects ever constructed in the world, and it plays a strategic role in solving the problem of uneven spatial and temporal distribution of water resources in China. The main canal is $1432 \mathrm{~km}$ long, the total amount of water to be diverted is 9.5 billion $\mathrm{m}^{3}$ per year, and the design discharge at the canal head is 350 $\mathrm{m}^{3} / \mathrm{s}$. There are more than 1800 hydraulic structures along the canal, including 64 check gates that divide the canal into 63 cascade pools, 97 diversion gates that transfer water to cities along the canal, and 54 drainage gates that abandon water in the case of an emergency. The cascade pools are operated and controlled to keep the downstream water levels of the pools constant. In general, the deviation of water levels should be small, and the drawdown rate should not exceed approximately $0.15 \mathrm{~m}$ in $1 \mathrm{~h}$ and $0.3 \mathrm{~m}$ in $24 \mathrm{~h}$. The quality of water to be diverted through the MRP should meet the Grade II

${ }^{a}$ Corresponding author: zhenghezhen@ $c j w s j y . c o m . c n$ 
standard of the Chinese Environmental Quality Standards for Surface Water (GB3838-2002) [11].

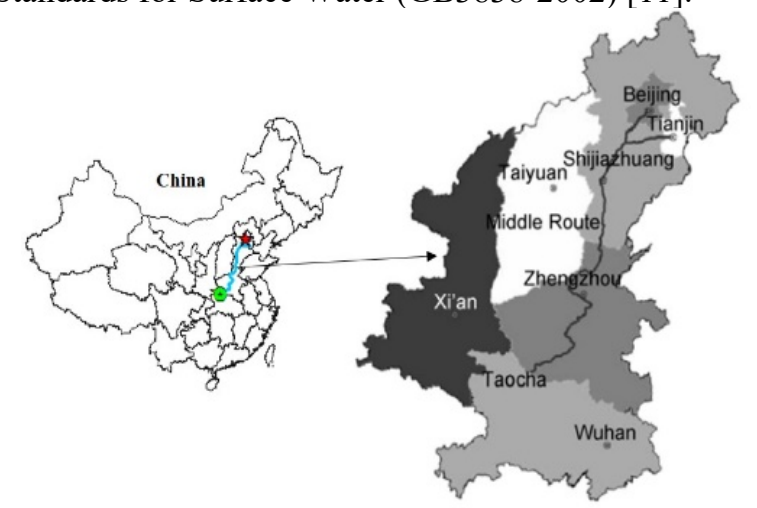

Figure 1. Diagram of the MRP.

\section{Emergency operation model}

If serious emergency accident (e.g., sudden water pollution accidents) occurs, the two check gates ( $\mathrm{K}$ and $\mathrm{K}+1$ ) of the accident pool need to be closed quickly (Figure 2). After check gate $\mathrm{K}$ is closed, the diversion gates in the upstream pools still need to maintain the initial outflows, while upstream check gates need to be closed to the final discharge. When emergency operation is taken, the discharge of upstream pools would decrease, if maintaining constant downstream water levels in upstream pools in the final state, the volume in the upstream pools should be reduced and surplus volume can only be abandoned. This model allows downstream levels to rise by a certain amount in the final state of emergency operation, and can minimize the total abandoned water through allocating volumes in the upstream pools.

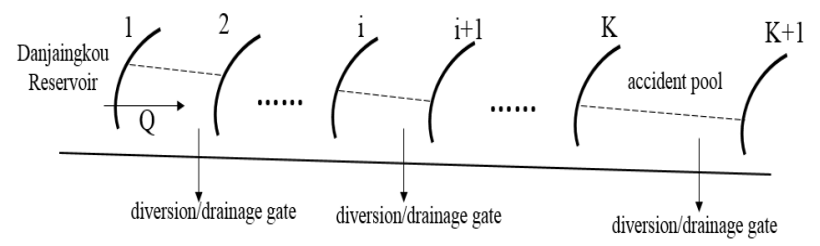

Figure 2. Diagram of upstream pools.

\subsection{Objective}

The objective is to minimize the total abandoned water during emergency operation process:

$$
\min W=\sum_{i=1}^{K-1} W_{i}
$$

Where $\mathrm{W}$ is the total abandoned water; $\mathrm{W}_{i}$ is the $i^{\text {th }}$ pool's abandoned water.

\subsection{Constraint conditions}

The constraint conditions are as follows:

1) Rises of upstream water levels of check gates in the final state are not over the maximum values:

$$
\Delta h_{i} \leq \Delta H_{i}
$$

Where $\Delta h_{i}$ and $\Delta H_{i}$ are the actual rise and maximum rise of upstream water level of $i^{\text {th }}$ check gate in the final state, respectively $(i=2 \ldots \mathrm{K})$.

2) Water level fluctuations of upstream water levels of check gates are not over the allowable maximum values:

$$
\Delta h_{\max , i} \leq \Delta H_{\max , i}
$$

Where $\Delta h_{\max , i}$ and $\Delta H_{\text {max }, i}$ are the actual and allowable maximum water level fluctuation of the $i^{\text {th }}$ check gate, respectively.

3) Each pool satisfies the discharge balance:

$$
Q_{u p, i}=Q_{d o w n, i}+Q_{d, i}
$$

Where $Q_{u p, i}, Q_{d o w n, i}$ and $Q_{d, i}$ are the discharges through the upstream check gate, downstream check gate and diversion gate of the $i^{\text {th }}$ pool, respectively $(i=1,2, \ldots, \mathrm{K}$ 1).

4) Each pool satisfies the water balance:

$$
V_{1, i}=V_{0, i}+V_{u p, i}-V_{d o w n, i}-V_{d, i}-V_{e, i}
$$

Where $V_{0, i}$ and $V_{1, i}$ are the initial and final volumes of the $i^{\text {th }}$ pool, respectively; $V_{u p, i}$ and $V_{\text {down,i }}$ are the amount flowing in and out of the $i^{\text {th }}$ pool through the check gates, respectively; $V_{d, i}$ and $V_{e, i}$ are the diverted and abandoned amount of the $i^{\text {th }}$ pool across the diversion gate and drainage gate, respectively $(i=1,2, \ldots, \mathrm{K}-1)$.

\subsection{Solution}

In the initial state, the discharges and upstream water levels of check gates, and discharges of diversion gates are known. In the final state, discharges of diversion gates in the upstream pools are unchanged, and discharges of check gates can easily be determined. Besides, the maximum rise of upstream water levels of check gates in the final state are pre-defined. Model solution steps are as follows:

Step 1: the initial volume $V_{0, i}$ and final maximum volume $V_{\max , i}(i=1,2 \ldots \mathrm{K}-1)$ in the upstream pools were calculated using the hydraulic model we developed [10];

Step 2: the differential between the initial volume and final maximum volume in each pool was calculated:

$$
\Delta V_{i}=V_{0, i}-V_{\max , i}
$$

If $\Delta V_{i}>0$, which indicates that the $i^{\text {th }}$ pool needs to abandon water out of itself or drain water into the downstream pool;

If $\Delta V_{i}=0$, which indicates that the $i^{\text {th }}$ pool doesn't need to abandon water nor need to receive drainage from the upstream pool; 
If $\Delta V_{i}<0$, which indicates that the $i^{\text {th }}$ pool can receive drainage from the upstream pool.

Step 3: the spatial relation about weather each pool need to abandon water or receive drainage is analyzed:

If each pool satisfies $\Delta V_{i}>0$, then each pool needs to abandon water and volume is $\Delta V_{i}$;

If each pool satisfies $\Delta V_{i} \leq 0$, then each pool doesn't abandon water;

If $\Delta V_{i}>0$ and $\Delta V_{i}<0$ are co-existed in upstream pools, the decomposition-coordination model [12] would be used to optimize volumes allocation in upstream pools.

The decomposition-coordination model can be divided into three layers:

The first layer: dividing the upstream pools into subsections:

If the first pool satisfies $\Delta V_{i}>0$, then the $m^{\text {th }}$ subsection is from the $m^{\text {th }}$ pool which satisfies $\Delta V_{i}>0$ to the $m+1^{\text {th }}$ pool which satisfies $\Delta V_{i}>0$. If the two pools are neighbours, they can be treated as one pool;

If the first pool satisfies $\Delta V_{i} \leq 0$, then the first subsection ranges from the first pool to the first pool which satisfies $\Delta V_{i}>0$, and the $m+1^{\text {th }}$ sub-section is from the $m^{\text {th }}$ pool satisfying $\Delta V_{i}>0$ to the $m+1^{\text {th }}$ pool satisfying $\Delta V_{i}>0$.

The second layer: volumes are allocated in each subsection. The sum of differentials (i.e., $\Delta V_{i}>0$ ) in the $m^{\text {th }}$ sub-section is:

$$
\Delta V_{m}=\sum \Delta V_{i}
$$

If $\Delta V_{m}>0$, which indicates that after volumes allocation (i.e. volume in each pool of this sub-section would be maximum in the final state), this sub-section still needs to abandon water out of itself or drain into the downstream sub-section, the amount is $\Delta V_{m}$;

If $\Delta V_{m}=0$, which indicates that each pool of this sub-section can be just fully stored according to the final maximum;

If $\Delta V_{m}<0$, which indicates that after volumes allocation, each pool of this sub-section can receive drainage from upstream pool and the amount is $\Delta V_{m}$.

The third layer: volumes are allocated between each sub-section.

If each sub-section satisfies $\Delta V_{m}>0$, which indicates volume in each pool of this sub-section would be maximum in the final state, and the surplus water $\Delta V_{m}$ will be abandoned out of each sub-section;

If each sub-section satisfies $\Delta V_{m} \leq 0$, which indicates each sub-section only needs to allocate water in its own pools;

If $\Delta V_{m}>0$ and $\Delta V_{m}<0$ are co-existed in the subsections, which indicates water between each sub-section need to be allocated: from the $m^{\text {th }}$ sub-section which satisfies $\Delta V_{m}>0$, add $\Delta V_{m}$ into the $m+1^{\text {th }}$ pool to continue next round of calculation.

\subsection{Allocation rules}

Water can only be drained out of itself or flow into the downstream pool, therefore the following rules are determined:

1) Rules of abandoning water out of the pool: if there is any drainage gate in the pool, water will be drained out through the drainage gate; otherwise water will be drained into the downstream pool and then abandoned out through the drainage gate in the downstream pool.

2) Rules of draining water into the downstream pool: if a pool needs to drain water into the downstream pool, then water can be drained according to the initial discharge of the downstream check gate, then the discharge of the check gate should be adjusted to the final value according to emergency operation requirement.

3) Rules of allocating water between the pools: if the surplus water of upstream pools needs to allocate, in order to simplify gate operation process, the downstream pool should receive the maximum amount according to the spatial orders.

\subsection{Gate operation}

Volume allocation between upstream pools can be determined by the above solution process, and the emergency operation measure of check gate $\mathrm{K}$ is predetermined, therefore, starting from the $\mathrm{K}-1^{\text {th }}$ pool to the first pool, the emergency operation measures of check gate and drainage gate in each pool can be determined step by step.

\section{Conclusions}

In order to save water resources, the emergency operation model in upstream pools of the accident pool of the MRP was built, which allows downstream levels to rise by a certain amount in the final state of emergency operation, and can minimize the total abandoned water through allocating volumes in the upstream pools.

Once emergency accident occurs in the MRP, this model would play an important role to determine reasonable emergency operation measures in upstream pools of the accident pool. In the future, the applications of this model and how to recover normal water supply after emergency operation will be deeply researched.

\section{Acknowledgements}

This paper was jointly supported by the Major Science and Technology Program for Water Pollution Control and Treatment (2017ZX07108-001), State Key Laboratory of Simulation and Regulation of Water Cycle in River Basin (2016CG05). 
Conflicts of Interest: The authors declare no conflict of interest.

\section{References}

1. R.Q. Grafton, J. Pittock, R. Davis, et al. Nature Clim. Change, 3:315-321 (2013)

2. Q.S. Qiao, K.L. Yang. J. Irrig. Drain Eng., 136(6): 383-391 (2010)

3. G. Liu. Wuhan University, (2013) (In Chinese)

4. Y.S. Liang, W. Wang, H.J. Li, X.H. Shen, Y.L. Xu, J.R. Dai. Parasite.Vectors., 5:52 (2012)

5. C. Tang, Y.Yi, Z. Yang, X. Cheng. J. Hydrol., 519: 2111-2120 (2014)

6. Y. Long, G. Xu, C. Ma. Environ. Sci. Pollut. R., 23 (12):12332-12342 (2016)

7. Y. Nie. Changjiang River Scientific Research Institute, (2011) (In Chinese)

8. X. Chen. China institute of Water Resources and Hydropower Research, (2015) (In Chinese)

9. A.J. Clemmens, R.J. Strand. J. Irrig. Drain Eng., 136(7): 460-469 (2010)

10. X. Lei, H. Zheng, Y. Shang, H. Wang. Int. J. Water Resour. D., 34 (3): 405-417 (2018)

11. X. Tang, M. Wu, W. Yang, W. Yin, F. Jin, M. Ye, N. Currie, M. Scholz. Water Air Soil Poll., 223:723737 (2012)

12. M.K. Kim, D. Hur. Electr. Power Energ. Syst., 33: 1638-1647 (2011) 